\title{
Continuous intracranial pressure monitoring via the shunt reservoir to assess suspected shunt malfunction in adults with hydrocephalus
}

\author{
Romergryko G. Geocadin, M.D., ${ }^{1,2}$ Panayiotis N. Varelas, M.D., Ph.D., ${ }^{4}$ \\ Daniele Rigamonti, M.D., ,,3 AND Michael A. Williams, M.D. ${ }^{1,2,3}$ \\ Departments of ${ }^{1}$ Neurology and ${ }^{2}$ Neurosurgery, and ${ }^{3}$ Adult Hydrocephalus Program, \\ Johns Hopkins Medical Institutions, Baltimore, Maryland; and ${ }^{4}$ Department of Neurology, \\ Henry Ford Hospital, Detroit, Michigan
}

\begin{abstract}
Object. The authors attempted to determine whether continuous intracrnial pressure monitoring via the shunt resevoir identifies ventriculoperitoneal (VP) shunt malfunctions that are not identified by radionuclide shunt patency study or shunt tap in adults with hydrocephalus.

Methods. During a 2-year period, 26 adults underwent 32 in-hospital continuous intracranial pressure (ICP) monitoring evaluations via needle access of a shunt reservoir. Monitoring was performed for $26.8 \pm 13.8$ hours (mean \pm standard deviation). No ICP waveform abnormality was detected in $31 \%$ of the evaluations (10 of 32 ). In contrast, abnormalities were detected in $69 \%$ ( 22 of 32 evaluations), including B waves (nine of 22 evaluations), siphoning (nine of 22 evaluations), and variable ICP (two of 22 evaluations). In 20 (91\%) of these 22 evaluations, the ICP abnormality was detected only after continuous ICP monitoring; in the other two evaluations, ICP became abnormal immediately on accessing the shunt reservoir. On the basis of the ICP monitoring results, shunt revision was performed in $66 \%$ (21 of 32 evaluations) and medical therapy was administered in $34 \%$ (11 of 32 evaluations). Shunt revision led to symptom improvement in 82\% (18 of 22 patients) and no change in $18 \%$ (four of 22 patients); medical therapy led to improvement in $18 \%$ (two of 11 patients), worsening in $18 \%$ (two of 11 patients), and no change in $64 \%$ (seven of 11 patients; $p<0.05$ ).

Conclusions. Continuous ICP monitoring via the shunt reservoir provides a more accurate assessment of shunt malfunction than transient ICP monitoring with a shunt tap or a radionuclide shunt patency study. It is a safe method for evaluating patients with suspected VP shunt malfunction, provides in vivo assessment of the effect of the shunt system on a patient's ICP, and can lead to more effective shunt revision.
\end{abstract}

KeY Words • hydrocephalus • siphoning • shunt malfunction • cerebrospinal fluid shunt • intracranial pressure

$\mathrm{H}$ YDROCEPHALUS is a disorder with ventriculomegaly caused by impairment of CSF circulation. Cerebrospinal fluid shunting is the most common treatment for hydrocephalus, and the VP shunt is the most common type of shunt configuration. Shunting CSF leads to improvement in patients with NPH; however, clinical improvement due to shunting has been varied. . $^{1,3,4,13,14}$ Failure to improve after CSF shunting has been attributed to complications such as obstruction, infection, and shunt malfunction. ${ }^{15}$ Another potential reason for failure to improve after shunting is a mismatch between the function of the shunt system and the patient's needs. Implantation of

Abbreviations used in this paper: $\mathrm{CSF}=$ cerebrospinal fluid; $\mathrm{ICP}=$ intracranial pressure; $\mathrm{NPH}=$ normal-pressure hydrocephalus; VP = ventriculoperitoneal. an unsuitable shunt can lead to overdrainage, underdrainage, or variable drainage of the CSF. We present our experience with ICP monitoring via the shunt reservoir to identify the type of shunt mismatch in a series of patients with hydrocephalus who had failed to improve after shunt surgery.

\section{CLINICAL MATERIAL AND METHODS}

This study included consecutive adult patients (18 years and older) with hydrocephalus and a VP shunt who had been referred to the Johns Hopkins Hospital over a 2-year period for evaluation of suspected shunt malfunction. The patients had been initially evaluated in the outpatient clinic by one of us (M.A.W.). The following preadmission data were collected: patient age and sex, initial disease 


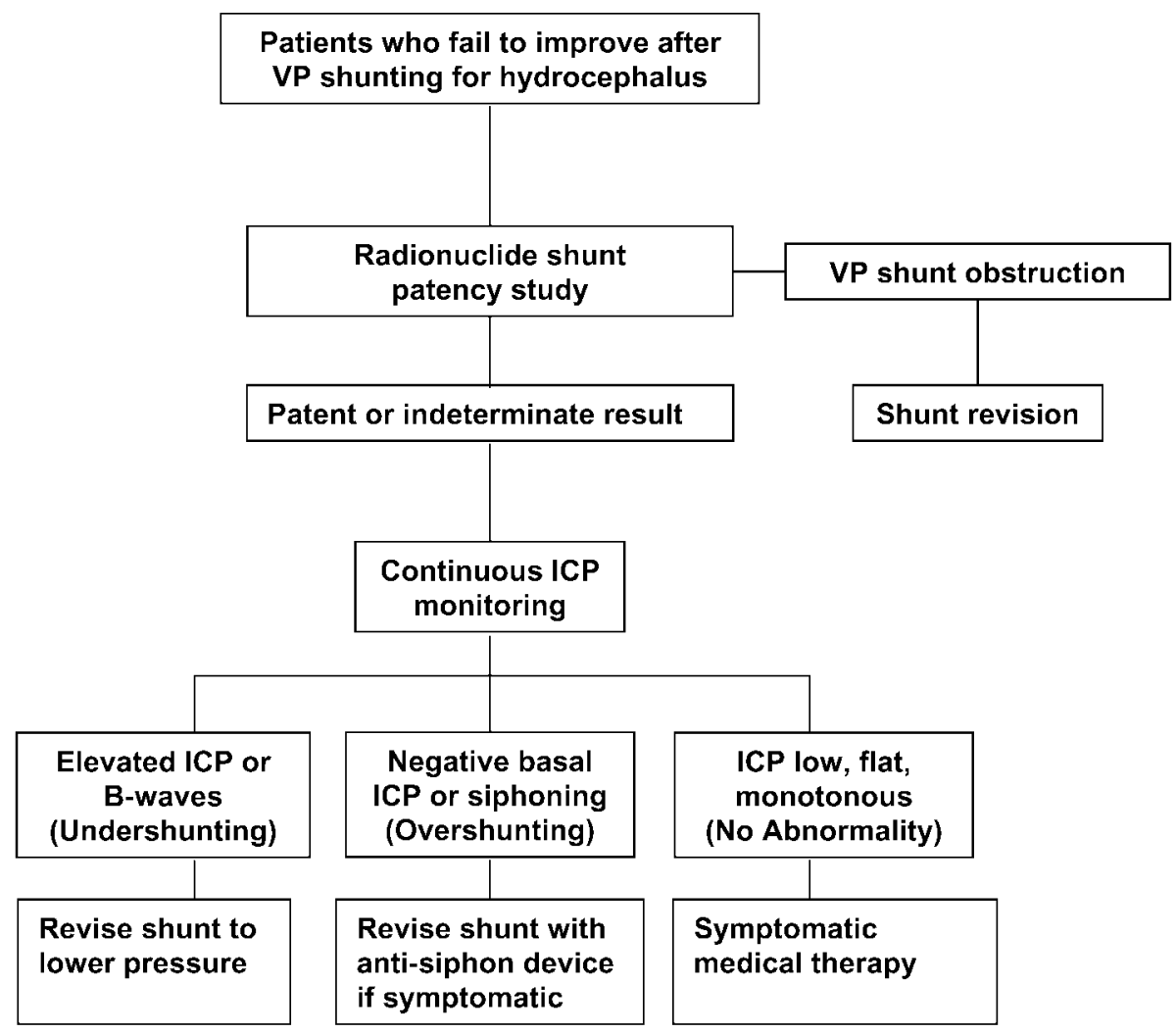

FIG. 1. The diagnostic algorithm used for evaluating patients who fail to improve after VP shunting for hydrocephalus.

leading to VP shunting, type of VP shunt used, most prominent symptoms after VP shunting, and radionuclide shunt patency study results. The algorithm for the diagnostic workup is presented in Fig. 1. Informed consent for all procedures was obtained from the patients or their surrogates. The diagnostic procedures were performed as part of our routine VP shunt evaluation protocol and were not regarded as investigational. The Johns Hopkins institutional review board approved the retrospective portion of this study.

Patients with suspected shunt malfunction were admitted electively to the hospital for 1 to 2 nights of continuous ICP monitoring via the shunt reservoir. Monitoring was terminated early if the results after the 1st night were considered diagnostic.

The scalp overlying the shunt was shaved, and a 25gauge butterfly needle (Abbott Laboratory) primed with nonbacteriostatic saline was inserted into the reservoir dome of the VP shunt system using an aseptic technique. ${ }^{5,9}$ The needle was secured to the scalp with sterile adhesive tape (Steri-Strip, 3 M Corp) and then covered with a protective cup that was secured to the head with gauze bandages. This setup allowed patients to move freely in bed without dislodging the butterfly needle. One dose of intravenous antibiotics was administered before the procedure (oxacillin, clindamycin, or vancomycin, depending on patient allergies). Prophylactic antibiotics were continued for the duration of ICP monitoring.

Intracranial pressure was measured using a standard fluid-coupled pressure transducer (Sorensen Custom Product, Abbott Critical Care System) and was displayed on the intensive care unit monitor (Marquette Tramscope 12E, Marquette Medical Systems, Inc.). The transducer was levelled with the patient's external acoustic meatus and was briefly opened to the atmosphere periodically throughout the recording period to document atmospheric zero reference pressure. Analog output from the intensive care unit monitor was transferred to a personal computer via a commercially available analog-to-digital signal converter (MacLab and PowerLab, AD Instruments). The digital display was analyzed in real time and saved for later offline analysis. All data were sampled at $40 \mathrm{~Hz}$. The ICP response to bilateral jugular vein compression was used to confirm that the pressure waveform reflected the ICP and not the intraabdominal pressure because of incorrect positioning of the needle in the reservoir.

The initial baseline ICP was recorded immediately on accessing the shunt reservoir to document the pressure reading that would be found on a routine shunt tap. Continuous ICP recordings were performed while the patient was at rest and during sleep to detect ICP waveforms such as A and B waves, ${ }^{11}$ and ICP was recorded with changes in the patient's position to detect siphoning. ${ }^{8}$ The A waves, also called "plateau waves," were defined as those produced during rapid ICP elevation to 50 to $100 \mathrm{~mm} \mathrm{Hg}$ lasting 5 to 20 minutes. The $\mathrm{B}$ waves were defined as those produced during rhythmic or semirhythmic ICP elevations with a peak systolic pressure at least $3 \mathrm{~mm} \mathrm{Hg}$ above the basal systolic pressure occurring at 0.5 to 2 cycles/ minute. ${ }^{11}$ Periods of quiet activity or sleep were indicated by the absence of a movement artifact on the recording and were annotated by the nursing staff. Except for peri- 
ods when the patient was directly observed for measurement of postural ICP changes, periods of wakefulness were not analyzed because the presence of movement artifacts obscured the waveforms. The duration of ICP monitoring was defined as the period during which ICP was continuously recorded.

For postural ICP monitoring, to record immediate changes in pressure and to allow for patient mobility during postural change, the transducer was attached to the head dressing just anterior to the ear and secured with tape. The construction of the transducer prevented placement of the zero reference point exactly at the external acoustic meatus. The level of the brow was the nearest stable position that could be used securely. Given that the vertical distance between the brow and the external acoustic meatus is generally $1 \mathrm{~cm}$ or less and that the horizontal distance was also $1 \mathrm{~cm}$ or less, we considered the measurement error associated with this position as negligible in comparison with the ICP abnormalities being examined.

We divided the patients into four groups based on the findings noted during the continuous ICP monitoring, namely, undershunting, overshunting, variable shunting, and no abnormality. We defined undershunting as either a sustained elevated resting ICP $(>15 \mathrm{~mm} \mathrm{Hg})$ or the presence of abnormal ICP waveforms. We defined overshunting as ICP that was low or negative, with a sustained resting ICP less than $0 \mathrm{~mm} \mathrm{Hg}$. Variable shunting was defined as the presence of both overshunting and undershunting.

The outcome of ICP monitoring led to shunt revision surgery in patients with abnormal ICP findings, and medical therapy (pharmacological headache treatment) in those with normal ICP findings. We selected the replacement valve for shunt revision on the basis of ICP monitoring results. For example, an antisiphon device was added if siphoning was present, or a lower-pressure valve was used if high pressure was present.

Patients underwent follow-up evaluation at least 1 month after the intervention. All patients had follow-up visits either with one of us (M.A.W.) or with their primary physician. We compared the symptom changes between patients treated with shunt revision and those treated with medical therapy based on the patient's report of significant improvement, worsening, or no change in relation to his or her baseline symptoms. Data are presented as the means \pm standard deviation or as a percentage.

\section{RESULTS}

There were 13 men and 13 women, with a mean age of $51.3 \pm 23.4$ years. Fourteen patients had obstructive hydrocephalus, and 12 had idiopathic NPH. The presenting symptoms that led to evaluation of the shunt are presented in Table 1. All patients had VP shunts. The types of VP shunts and ICP monitoring results are featured in Table 2. Eighteen patients underwent radionuclide shunt patency studies before the ICP monitoring. Radionuclide clearance was normal in 15 patients (83\%) and was delayed in three patients $(16.7 \%)$, suggesting partial obstruction.

Twenty-six patients underwent 32 evaluations with continuous ICP monitoring via the shunt reservoir. Four patients underwent two separate evaluations, and one underwent three. The mean duration of continuous monitoring
TABLE 1

Symptoms leading to ICP monitoring*

\begin{tabular}{lc}
\hline \multicolumn{1}{c}{ Symptoms } & No. of ICP Evaluations (\%) \\
\hline headache & $17(65)$ \\
worsening imbalance & $13(50)$ \\
worsening incontinence & $3(11.5)$ \\
dementia & $6(23)$ \\
tremor & $2(7.7)$ \\
\hline
\end{tabular}

* Some patients had more than one presenting symptom.

was $26.8 \pm 13.8$ hours. To access the reservoir with the needle, only one attempt was needed in 26 evaluations, two attempts in two evaluations, and four attempts in one patient because the needle dislodged repeatedly. Except for mild discomfort associated with needle insertion into the shunt reservoir, there were no complications related to the procedure.

Results of $69 \%$ of the ICP monitoring evaluations (22 of 32) were abnormal, and 31\% (10 of 32) showed no abnormality as defined a priori. The baseline ICP on initial monitoring of the four groups is shown in Fig. 2. Detailed results are presented in Table 2.

A critical finding is that only two (9\%) of 22 abnormal recordings on continuous monitoring were associated with an abnormal initial baseline ICP: one patient had elevated ICP of $25 \mathrm{~mm} \mathrm{Hg}$ and one had low ICP of $-10 \mathrm{~mm} \mathrm{Hg}$. In the remaining 20 patients $(91 \%)$, the ICP abnormalities were not apparent for several hours.

Of the 32 evaluations, $21(66 \%)$ led to surgical revision of the shunt, and 20 (95\%) of the 21 evaluations were abnormal. One patient with normal ICP was treated with surgical shunt revision because of previous dramatic symptom improvement with external CSF drainage via a spinal catheter. Of the remaining 11 evaluations, nine had normal, monotonous ICP patterns consistent with normal shunt function; two had abnormal ICP patterns, but the patients declined shunt revision surgery.

At the follow-up evaluation, patients who had undergone shunt revision surgery had a better overall outcome, with symptom improvement in $82 \%$ (18 of 22 patients) and no change in $18 \%$ (four of 22 patients). The use of medical therapy was followed by symptom improvement in $18 \%$ (two of 11 patients), no change in 64\% (seven of 11 patients), and worsening in $18 \%$ (two of 11 patients; $\mathrm{p}<$ $0.05)$.

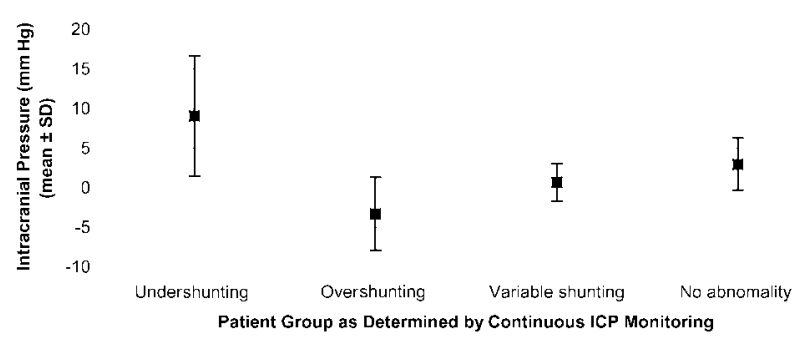

FIG. 2. Graph showing a comparison of the initial baseline ICP (as would be found at a shunt tap) for each patient group, as determined by continuous ICP monitoring. There is no statistical difference between groups. SD = standard deviation. 
TABLE 2

Ventriculoperitoneal shunts assessed in 26 patients with hydrocephalus

\begin{tabular}{|c|c|c|c|c|c|}
\hline \multirow[b]{2}{*}{$\begin{array}{c}\text { VP Shunt } \\
\text { (manufacturer) }\end{array}$} & \multicolumn{5}{|c|}{ No. of Patients (\%) } \\
\hline & Total & $\begin{array}{l}\text { Over- } \\
\text { shunting }\end{array}$ & $\begin{array}{l}\text { Under- } \\
\text { shunting }\end{array}$ & $\begin{array}{l}\text { Variable } \\
\text { Shunting }\end{array}$ & $\begin{array}{l}\text { Normal } \\
\text { ICP }\end{array}$ \\
\hline $\begin{array}{l}\text { PS Medical Delta } \\
\text { Valve (Medtronic) }\end{array}$ & $18(69.2)$ & 7 & 4 & 2 & 6 \\
\hline $\begin{array}{l}\text { Codman Hakim } \\
\text { Valve (Codman) }\end{array}$ & $3(11.5)$ & 0 & 2 & 1 & 0 \\
\hline $\begin{array}{l}\text { Cordis Orbis Sigma } \\
\text { Valve (Cordis) }\end{array}$ & $2(7.7)$ & 1 & 0 & 0 & 1 \\
\hline $\begin{array}{l}\text { Phoenix Valve (Phoenix } \\
\text { Biomedical, Inc.) }\end{array}$ & $1(3.8)$ & 1 & 0 & 0 & 0 \\
\hline undetermined & $2(7.7)$ & 0 & 1 & 0 & 1 \\
\hline
\end{tabular}

\section{DISCUSSION}

The goal of this study was to examine the role of continuous ICP monitoring in evaluating shunt malfunction in hydrocephalus in adults. Our data demonstrate that continuous monitoring provides a more accurate assessment of shunt function than transient ICP assessment with a shunt tap, a commonly used procedure in which a butterfly needle is inserted into the shunt reservoir and the height of the CSF in the tubing provides an estimate of the ICP at that moment by simple manometry. The initial baseline pressure was normal in $90 \%$ of the evaluations in which abnormal ICP patterns were subsequently identified with prolonged monitoring. Abnormal ICP findings, such as B waves, were evident only during sleep or quiet activity, and intracranial hypotension was observed only by having the patient sit or stand.

Thus, transient ICP assessment, as done with a shunt tap, has a high false-negative rate for assessing shunt malfunction. Its main limitation is that it demonstrates the ICP for only a few moments, whereas continuous monitoring reveals the full range of ICP variations during wakefulness, sleep, or change in patient position. For continuous ICP monitoring, because the needle, tubing, and transducer are closed to the atmosphere, it is possible to record subatmospheric pressure, or siphoning, without introducing air into the shunt, which is a risk with the shunt tap because the tubing is open to the atmosphere.

Shunt assessment by continuous ICP monitoring revealed shunt malfunction in patients whose radionuclide shunt patency study results were either indeterminate or suggested that the shunt was patent. This discrepancy highlights the fact that shunt patency studies only identify CSF flow through the shunt system and do not provide information about ICP or the differential pressure gradients that drive the flow. Our results demonstrated that a shunt found to be patent by radionuclide testing could still be malfunctioning, such as regulating the ICP too high for the patient's needs.

Continuous ICP monitoring identified the type of mismatch between the VP shunt and the ICP characteristics in the patient. The mismatch can appear in three ways: undershunting, overshunting, and variable shunting (both under- and overshunting). If the shunt opening pressure is above the patient's ICP, then the valve does not open and insufficient CSF is drained, leading to undershunting. This circumstance can lead to a recurrence of prior symptoms and will manifest on ICP monitoring as elevated basal ICP, B waves, or plateau waves. Abnormal ICP waveforms, especially plateau waves and B waves, reflect the loss of cranial vault compliance from any of a number of origins, and the purpose of a shunt is to correct cranial vault compliance by providing a route of egress for CSF. Thus, these waveforms should not be present if the shunt is functioning properly.

In cases in which the shunt opening pressure is too low for the patient's ICP needs, excessive CSF drainage leads to overshunting, or siphoning, which is abnormal gravityinduced flow through CSF shunts while the patient is upright. The associated intracranial hypotension can lead to disabling symptoms. ${ }^{8}$ Foltz and Blanks ${ }^{8}$ reported on a series of 14 patients who had previously undergone shunting for hydrocephalus and who had symptoms when upright and active that were usually relieved by lying down. Intracranial pressure was dramatically low, with a marked decrease when the patient assumed an upright position, and near normal levels when supine. Shunt revision led to immediate clinical improvement with normal ICP measurements. It is important to note, however, that some patients with hydrocephalus need very low CSF pressure for improvement. ${ }^{10}$ Bergsneider and colleagues ${ }^{2}$ showed that siphoning by external ventricular drainage together with a reduction in ventricle size resulted in symptomatic improvement in adults with hydrocephalus. Therefore, siphoning can be either harmful or beneficial to patients with hydrocephalus, depending on individual characteristics. It is important to identify these subsets of patients. We believe that continuous ICP monitoring guides therapeutic decisions in such patients. In those with symptoms of overshunting, we recommend either shunt revision with a higher shunt pressure or placement of an antisiphon device.

Last, a patent but incorrectly functioning shunt valve can cause erratic CSF drainage leading to variable ICP patterns (that is, overshunting and undershunting). In these cases, a lower shunt pressure was selected for the predominantly elevated ICP and a higher shunt pressure for those with predominantly low ICP.

Overall, $82 \%$ of the patients in the present study experienced symptomatic improvement after the shunts were revised on the basis of the ICP monitoring results. This result is at odds with those of Eide, ${ }^{6}$ who found that symptoms were unchanged in $58 \%$ of cases in which continuous ICP monitoring was used to guide clinical decision making. Although the patient populations in the Eide study and the present study appear similar, Eide measured ICP using a parenchymal ICP monitor, whereas we measured ICP by accessing the CSF in the shunt reservoir. Without a direct and simultaneous comparison of the methods, it is difficult to say whether the differences in the ICP assessment methods were related to the differences in patient outcomes.

Cerebrospinal fluid shunting in adults with hydrocephalus leads to a favorable outcome in 52.6 to $90 \%$ of patients. ${ }^{1,3,4,13,14}$ Whereas an incorrect diagnosis of NPH is one explanation for the lower response rates, another is shunt malfunction, which occurs in 10.6 to $31 \%$ of cases ${ }^{4,9}$ and would prevent clinical improvement. Despite the large 
number of patients who fail to respond to shunting, there is no effective and noninvasive way of evaluating these patients. Our method of continuous ICP monitoring allows an in vivo evaluation of the dynamic interaction between the shunt system and the patient during different physiological conditions, which leads to a better understanding of the causes of shunt malfunction and identifies patients with a shunt mismatch.

Except for the initial mild discomfort associated with the introduction of the needle into the shunt reservoir, all patients tolerated the ICP monitoring procedure well. We did not observe any complications related to the introduction of the needle into the shunt reservoir. There was no mechanical harm to the shunt. Although we did not obtain CSF for sampling, no patient had signs or symptoms of shunt infection.

Assessment of CSF outflow resistance $\left(\mathrm{R}_{\text {out }}\right)$ has been proposed as an alternative method of determining whether a shunt is functioning or malfunctioning. ${ }^{7,12}$ This method, which is virtually identical to the technique used to assess outflow resistance for the initial diagnosis of NPH, entails a lumbar puncture with two needles and requires special expertise and equipment that is not widely available. Another limitation of this method is that it cannot demonstrate whether intracranial hypotension (siphoning) is present.

The current study is limited because it includes only a select group of patients with symptoms suggestive of VP shunt malfunction or shunt mismatch. We focused on the alleviation of symptoms and offered surgical revision in cases of shunt malfunction or mismatch. It is presumed, but not demonstrated, that the abnormal ICP seen before shunt revision was absent in the patients who improved after revision. We did not directly perform the traditional shunt tap method of ICP measurement; however, we believe the assessment of the initial baseline ICP is equivalent because we used an isovolemic method (that is, no CSF loss or aspiration) to access the shunt reservoir. Another drawback of continuous ICP monitoring is the need for hospital admission in a closely observed setting. We believe that the hospital admission is warranted because continuous ICP monitoring will help determine the nature of the shunt malfunction and lead to goal-directed therapeutic decisions.

Most of the shunts assessed in this study were singlesetting shunts, although three of the shunts were the adjustable Codman Hakim shunt. Our study results led to shunt revision surgery in two thirds of all patients. We anticipate that the use of an adjustable shunt would reduce the need for revision surgery because the shunt could be adjusted to suit each patient's needs; however, adjustable shunts can still be susceptible to obstruction or to valve malfunction.

\section{CONCLUSIONS}

In summary, the study data demonstrate that continuous ICP monitoring can identify a significant number of shunt malfunctions not identified by either shunt tap or radionuclide shunt patency studies. Continuous ICP monitoring provides in vivo assessment of the dynamic interaction between the shunt system and the patient's ICP dynamics such as seen during rest and sleep or a change in posture.
The results of continuous ICP monitoring can be used to guide therapeutic decisions. Continuous ICP monitoring via the shunt reservoir is a safe procedure.

\section{Acknowledgment}

We acknowledge the physician and nursing staff of the NeuroProgressive Care Unit and Neurosciences Critical Care Unit of the Johns Hopkins Hospital for their diligent care of patients and invaluable support for the ICP evaluation protocols.

\section{Disclosure}

In the 12 months before the submission of this manuscript, Dr. Rigamonti has received support from Medtronic for research related to hydrocephalus in adults, including salary support.

In the 12 months before the submission of this manuscript, Dr. Williams has received honoraria from Medtronic for speaking on the topic of hydrocephalus in adults and has received support from Medtronic for research related to hydrocephalus in adults.

\section{References}

1. Bergsneider M, Black PM, Klinge P, Marmarou A, Relkin N: Surgical management of idiopathic normal-pressure hydrocephalus. Neurosurgery 57 (3 Suppl): S29-S39, 2005

2. Bergsneider M, Peacock WJ, Mazziotta JC, Becker DP: Beneficial effect of siphoning in treatment of adult hydrocephalus. Arch Neurol 56:1224-1229, 1999

3. Borgesen SE, Gjerris F, Sorensen SC: Intracranial pressure and conductance to outflow of cerebrospinal fluid in normal-pressure hydrocephalus. J Neurosurg 50:489-493, 1979

4. Bret P, Chazal J, Janny P, Renaud B, Tommasi M, Lemaire JJ, et al: [Chronic hydrocephalus in adults.] Neurochirurgie 36 (1 Suppl):1-159, 1990 (Fr)

5. Chhabra DK, Agrawal GD, Mittal P: "Z" flow hydrocephalus shunt, a new approach to the problem of hydrocephalus, the rationale behind its design and the initial results of pressure monitoring after "Z" flow shunt implantation. Acta Neurochir (Wien) 121:43-47, 1993

6. Eide PK: Quantitative analysis of continuous intracranial pressure recordings in symptomatic patients with extracranial shunts. J Neurol Neurosurg Psychiatry 74:231-237, 2003

7. Eklund A, Lundkvist B, Koskinen LO, Malm J: Infusion technique can be used to distinguish between dysfunction of a hydrocephalus shunt system and a progressive dementia. Med Biol Eng Comput 42:644-649, 2004

8. Foltz EL, Blanks JP: Symptomatic low intracranial pressure in shunted hydrocephalus. J Neurosurg 68:401-408, 1988

9. Larsson A, Wikkelso C, Bilting M, Stephensen H: Clinical parameters in 74 consecutive patients shunt operated for normal pressure hydrocephalus. Acta Neurol Scand 84:475-482, 1991

10. Lesniak M, Clatterbuck R, Rigamonti D, Williams MA: Low pressure hydrocephalus and ventriculomegaly: hysteresis, nonlinear dynamics, and the benefits of CSF diversion. Br J Neurosurg 16:555-561, 2002

11. Lundberg N: Continuous recording and control of ventricular fluid pressure in neurosurgical practice. Acta Psychiatr Neurol Scand 36:1-193, 1960

12. Malm J, Lundkvist B, Eklund A, Koskinen LO, Kristensen B: CSF outflow resistance as a predictor of shunt function. A longterm study. Acta Neurol Scand 110:154-160, 2004

13. Marmarou A, Young HF, Aygok GA, Sawauchi S, Tsuji O, Yamamoto T, et al: Diagnosis and management of idiopathic normal pressure hydrocephalus: a prospective study in $151 \mathrm{pa}-$ tients. J Neurosurg 102:987-997, 2005

14. McGirt MJ, Woodworth G, Coon AL, Thomas G, Williams MA, Rigamonti D: Diagnosis, treatment, and analysis of long- 
term outcomes in idiopathic normal-pressure hydrocephalus. Neurosurgery 57:699-705, 2005

15. Puca A, Anile C, Maira G, Rossi G: Cerebrospinal fluid shunting for hydrocephalus in the adult: factors related to shunt revision. Neurosurgery 29:822-826, 1991

Manuscript submitted January 15, 2007.

Accepted March 2, 2007.
This work was supported in part by the Eleanor Naylor Dana Neuroscience Critical Care Fellowship (R.G.G. and P.N.V.), the David S. Dana Research Prize (R.G.G.), and the Schoendorf Foundation (D.R. and M.A.W.)

Address reprint requests to: Michael A. Williams, M.D.., Johns Hopkins Adult Hydrocephalus Program, 600 North Wolfe Street, Phipps 100, Baltimore, Maryland 21287. email: mwilliam@jhmi.edu. 